\title{
Expanding role of lenalidomide in hematologic malignancies
}

This article was published in the following Dove Press journal:

Cancer Management and Research

2 May 2015

Number of times this article has been viewed

\section{Nilanjan Ghosh \\ Michael R Grunwald \\ Omotayo Fasan \\ Manisha Bhutani}

Levine Cancer Institute, Carolinas HealthCare System, Charlotte, NC, USA

Video abstract

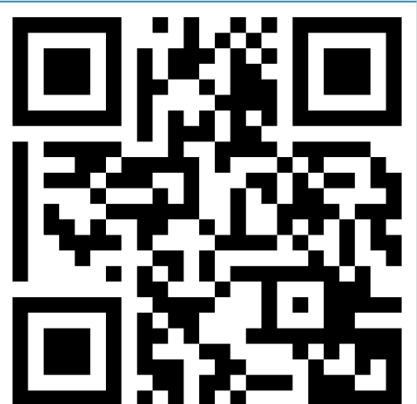

Point your SmartPhone at the code above. If you have a QR code reader the video abstract will appear. Or use: http://youtu.be/s8P L-OLiks
Correspondence: Nilanjan Ghosh Department of Hematologic Oncology and Blood Disorders, Levine Cancer Institute, 1021 Morehead Medical Drive, Suite 5300, Charlotte, NC 28204, USA

$\mathrm{Tel}+\mathrm{I} 9804424363$

Fax + I 980442 524I

Email nilanjan.ghosh@carolinashealthcare. org
Abstract: Lenalidomide is an immunomodulatory agent that has been approved by the US Food and Drug Administration for treatment of multiple myeloma, deletion 5q myelodysplastic syndrome, and mantle cell lymphoma. In addition, it has clinical activity in lymphoproliferative disorders and acute myeloid leukemia. The mode of action includes immunomodulatory, anti-inflammatory, antiangiogenic, and antiproliferative mechanisms. The antitumor effect is a result of direct interference of key pathways in tumor cells and indirect modulation of the tumor microenvironment. There has been no recent collective review on lenalidomide in multiple myeloma, myelodysplastic syndrome/acute myeloid leukemia, and lymphoma. This review summarizes the results of current clinical studies of lenalidomide, alone and in combination with other agents, as a therapeutic option for various hematologic malignancies.

Keywords: IMiD, leukemia, lymphoma, myeloma, MDS

\section{Introduction}

Lenalidomide and pomalidomide are immunomodulatory drugs (IMiDs) that have been derived by altering the structure of the parent drug: thalidomide. In the late 1990s, thalidomide was found to be effective in multiple myeloma (MM) when it was tested for its antiangiogenic potential. Subsequent efforts to improve the efficacy and reduce the toxicity of this agent led to the development of lenalidomide. Several mechanisms have been proposed by which lenalidomide has shown to boost immune responses. Lenalidomide enhances antigen uptake by antigen-presenting cells ${ }^{1}$ and potentiates immune responses by restoring dendritic cell function, inhibiting regulatory T-cell activity, and activating NK cells and T-cells by boosting production of interferon gamma and interleukin-2.,3 The IMiDs possess antiangiogenic properties; ${ }^{4,5}$ impair interaction between malignant cells and bone marrow stromal cells; $;{ }^{6}$ induce cell cycle arrest; and have direct antiproliferative effects. ${ }^{7,8}$ However, the biochemical mechanisms underlying their therapeutic effects were not known until recently.

Thalidomide binds cereblon, a substrate-recognition component of an ubiquitin ligase, and inhibits autoubiquitination. ${ }^{9}$ In MM as well as activated B-cell type (ABC) diffuse large B-cell lymphoma, cereblon has been identified as the target for the immunomodulatory and antiproliferative activities of lenalidomide. ${ }^{10,11}$ IMiD resistance is associated with downregulation of cereblon ${ }^{12}$ and high concentrations of cereblon are associated with increased responsiveness to IMiDs. ${ }^{13}$ In MM cells, lenalidomidebound cereblon acquires the ability to target the proteosomal degradation of two B-cell transcription factors, IKZF1 and IKZF3, an essential step in the anti-myeloma effect. ${ }^{14}$ In ABC diffuse large B-cell lymphoma, the tumoricidal effect of lenalidomide 
is associated with the cereblon-mediated downregulation of IRF-4 leading to inhibition of the B-cell receptor-NF-kB signaling pathway. ${ }^{11}$ In myelodysplastic syndrome (MDS) with 5q-, a novel cereblon substrate, casein kinase 1A1 (CSNK1A1) has been identified as a lenalidomide target; increased ubiquitination and decreased levels of CSNK1A1 have been noted with lenalidomide treatment in 5q-MDS. ${ }^{15}$ The proposed mechanisms of actions of lenalidomide are mainly supported by in vitro studies and some ex vivo studies, although the clear evidence attesting to these mechanistic links in various clinical settings remains to be systematically explored. For instance, concurrent administration of dexamethasone with lenalidomide in MM patients clearly improves efficacy irrespective of the fact that dexamethasone is antagonistic to lenalidomide's immune-stimulatory effects on NK cells and T regulatory cells. ${ }^{16}$ Hence, more work is required to understand whether the clinical efficacy of lenalidomide in hematologic malignancies is linked to a common target or represents summation of several independent effects.

\section{Chemical structure and pharmacokinetics}

Lenalidomide was the first analog of thalidomide. The International Union of Pure and Applied Chemistry name for the compound is 3-(4-amino-1-oxo-1,3-dihydro-2Hisoindol-2-yl)piperidine-2-6-dione. The molecular formula for lenalidomide is $\mathrm{C} 13 \mathrm{H} 13 \mathrm{~N} 3 \mathrm{O} 3$. It is structurally similar to thalidomide, but it possesses an asymmetric carbon atom and therefore can exist in the optically active enantiomers $\mathrm{S}(-)$ and $\mathrm{R}(+)$. It is produced as a racemic mixture with a net optical rotation of zero. The solubility in organic solvents is greater than in aqueous solvents. In vitro anti-inflammatory studies reveal lenalidomide to be about 1,000-times more potent than thalidomide whereas pomalidomide is ten-times more potent than lenalidomide. However the maximum tolerated dose and plasma levels of pomalidomide are at least ten-times lower than for lenalidomide, hence this higher potency of pomalidomide may not be clinically exploitable.

The development of lenalidomide was spurred by the need to reduce the nonhematologic adverse effects of thalidomide and to enhance the immunomodulatory effects; the compound was rationally designed to achieve these goals. ${ }^{17,18}$ The chemical structure imparts less sedation, constipation, and neuropathy compared to thalidomide. However, it is more myelosuppressive and the incidence of grade 3 /grade 4 hematologic toxicities are leukopenia: 5\%-7\%, anemia: $6 \%-11 \%$, and thrombocytopenia: $12 \%-50 \%$.
Lenalidomide is rapidly absorbed from the gastrointestinal tract with peak levels achieved in 30-360 minutes. The protein binding of lenalidomide at $30 \%$ is less than of thalidomide at $55 \%-65 \%$. It has a shorter elimination half-life of $\sim 3$ hours compared to thalidomide at $\sim 5.5$ hours and pomalidomide at $\sim 7.5$ hours. It is excreted in the urine mostly as unchanged drug (82\%); dialysis is able to remove $40 \%$ of a dose.

\section{Clinical utility in hematologic malignancies MM}

The use of IMiDs and proteasome inhibitors together with advances in autologous stem-cell transplantation (ASCT) have improved survival substantially in MM patients. Lenalidomide provides rapid and sustained disease control and has been integrated into therapy for relapsed/refractory and newly diagnosed transplant-eligible and -ineligible MM patients in all disease settings. The earliest Phase I/II trials, which were reported more than 10 years ago, demonstrated manageable toxicity with promising anti-MM activity. The reports highlighted that lenalidomide lacked the adverse effects associated with thalidomide, namely a high incidence of peripheral neuropathy, constipation, and somnolence. ${ }^{19}$ Based on these results, lenalidomide was granted orphan status in 2001 and fast-track status in 2003, which led to the opening of two large Phase III randomized placebo-controlled registration trials (MM-009 in US and Canada, and MM-010 in Europe, Australia, and Israel) for relapsed/refractory MM. These trials compared lenalidomide plus dexamethasone (RD) to dexamethasone alone and enrolled 353 and 351 patients, respectively $(\mathrm{n}=704) .{ }^{20,21}$ In both arms, pulsed high-dose dexamethasone (480 $\mathrm{mg}$ per cycle) was used in 28-day cycles, and treatment was continued until the disease progressed or unacceptable adverse effects occurred. Both studies were stopped at the first preplanned interim analysis for benefit and demonstrated superiority of RD compared with single-agent dexamethasone in terms of response rates, time to progression, and overall survival (OS). Mature pooled data of these two studies at a median follow-up of 4 years showed a sustained significant benefit for the lenalidomide arm (median OS of 38 months compared with 31.6 months, respectively; $P=0.045) .{ }^{22}$ Based on this data, lenalidomide (in combination with dexamethasone) was approved by the US Food and Drug Administration (FDA) in 2006 for the treatment of relapsed/refractory MM patients who have received at least one prior line of therapy. The efficacy of lenalidomide has been confirmed as well in the previously 
untreated population (both transplant eligible and ineligible) and as maintenance therapy following ASCT or following non-transplant induction regimens (Table 1). ${ }^{20-29}$

Lenalidomide is effective and well tolerated when used as part of first-line regimens in previously untreated patients. Zonder et al showed an improved median 1-year progressionfree survival (PFS) (78\% versus 52\%) and improved OS (77\% versus $48 \%$ ) in patients receiving RD compared with placebo/dexamethasone. ${ }^{24}$ The improved safety profile and lower rate of early deaths associated with low-dose dexamethasone (160 mg per cycle) plus lenalidomide ( $R d)$ versus $\mathrm{RD}$ have led to widespread adoption of this approach as initial therapy for both transplant-eligible and transplant-ineligible patients. ${ }^{25}$ In the recently reported randomized Phase III Frontline Investigation of REVLIMID + Dexamethasone Versus Standard Thalidomide (FIRST) trial in elderly patients with previously untreated MM not eligible for transplantation, a continuous regimen of $\mathrm{Rd}$ emerged as the winner with median progression free survival of 25.5 months when compared with 20.7 months for a fixed course (18 cycles) of Rd (Rd18) and 21.2 months for melphalan, prednisone, and thalidomide (MPT) ${ }^{26}$ This study demonstrated a $28 \%$ reduction in risk of progression or death for patients treated with continuous $\mathrm{Rd}$ administered until progression compared with those treated with MPT (hazard ratio [HR] 0.72; $P<0.001$ ) and a $30 \%$ risk reduction compared with $\mathrm{Rd} 18$ (HR 0.70; $P<0.001)$. Additionally, response rates were significantly higher with continuous $\operatorname{Rd}(75 \%)$ and with $\operatorname{Rd} 18$ (73\%) than with MPT ( $62 \% ; P<0.001$ for both comparisons).

The $\mathrm{Rd}$ regimen is also the backbone upon which numerous other regimens are built, for example, cyclophosphamide/ $\mathrm{Rd}$, clarithromycin (Biaxin)/Rd, bortezomib (Velcade)/Rd (VRd), pegylated doxorubicin/Rd, carfilzomib (Kyprolis)/Rd (KRd), ixazomib/Rd, etc Limited data are available to draw conclusions regarding the superiority of one lenalidomidebased combination over another. Response rates are clearly

Table I Key Phase III randomized clinical trials in MM incorporating lenalidomide

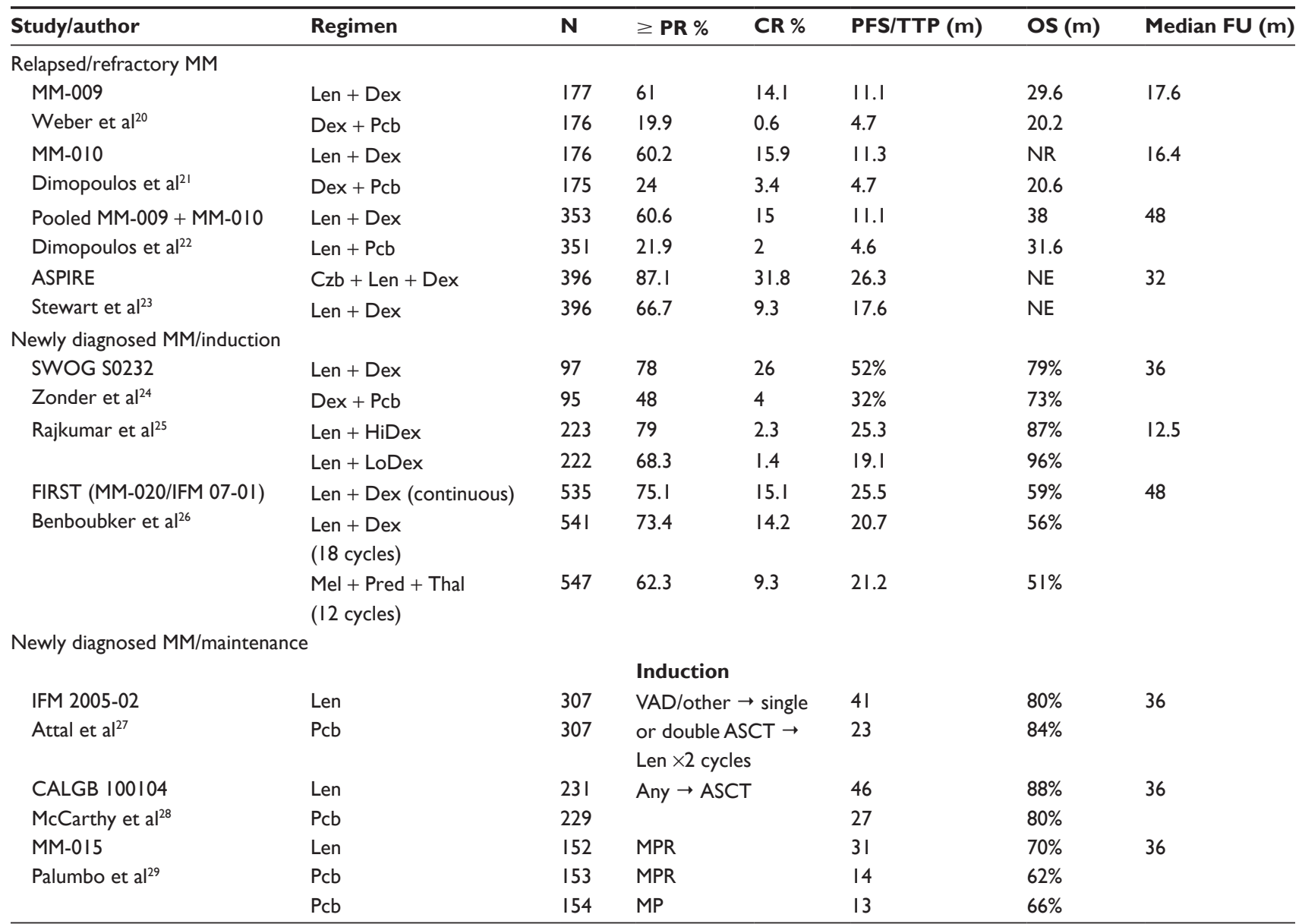

Abbreviations: ASCT, autologous stem cell transplant; CR, complete response; Czb, carfilzomib; Dex, dexamethasone; FU, follow-up; HiDex, high-dose dexamethasone; Len, lenalidomide; LoDex, low-dose dexamethasone; m, months; MM, multiple myeloma; Mel, melphalan; MP, melphalan-prednisone; MPR, melphalan-prednisone-lenalidomide; NE, not evaluable; NR, not reported; OS, overall survival; Pcb, placebo; PFS, progression-free survival; PR, partial response; Pred, prednisone; Thal, thalidomide; TTP, time to progression; VAD, vincristine-doxorubicin-dexamethasone. 
improved with three-drug regimens, although demonstration of a significant survival advantage is difficult given the large numbers of patients and the long duration of follow-up required, and the availability of effective salvage therapies. Thus, based on response rates and depth of response as surrogate markers for outcome, VRd is commonly included in myeloma treatment guidelines as one of several preferred options for initial therapy for transplant-eligible as well as for transplant-ineligible patients in the United States. VRd is currently being evaluated as frontline therapy in several Phase III trials. Specifically trials in the United States are comparing VRd with Rd, VRd with KRd, as well as VRd with and without stem cell transplant. The results of a recently published randomized trial comparing KRd with Rd expand the evidence supporting use of a three-drug regimen in patients with relapsed MM. In this trial, the addition of carfilzomib to $\mathrm{Rd}$ resulted in significant improvement in PFS (median, 26.3 months versus 17.6 months in the Rd group; HR 0.69 ; $P=0.0001)$ at the interim analysis and had a favorable riskbenefit profile. ${ }^{23} \mathrm{~A}$ wide range of novel combination regimens built on the Rd backbone are in development, aimed at further improving treatment options for patients with MM. Three monoclonal antibodies - daratumumab, SAR650984, and elotuzumab - are in various phases of clinical development and show encouraging activity when combined with $\mathrm{Rd}$ in MM.

In addition to being studied in induction regimens, lenalidomide has been investigated for use post-ASCT for consolidation and maintenance. Lenalidomide has been evaluated in three Phase III studies in patients as maintenance therapy following ASCT (CALGB 100104, IFM 2005-02) or as continuous therapy for transplant-ineligible patients (MM-015). ${ }^{27-29}$ All three trials reported significant progression free survival benefit with the use of lenalidomide maintenance compared with placebo, the CALGB study reported significant difference in 3-year OS $(88 \%$ versus $80 \%, P=0.03) .{ }^{28}$ Data on using lenalidomide as a maintenance strategy are less than clear as regards OS and further studies are awaited.

In newly diagnosed MM, lenalidomide is part of widely used triplet and doublet combinations such as VRd and Rd, respectively. There are no head-to-head comparisons between $\mathrm{Rd}$ and $\mathrm{Vd}$, or between VRd and cytoxan/bortezomib/ dexamethasone (CyBorD). The choice of using a lenalidomide-based regimen is often dictated by approval status of lenalidomide for initial therapy, drug availability, disease characteristics, patient preferences, existing comorbidities, and cost considerations. CyBorD is generally preferred for those with renal failure. $\mathrm{Rd}$ is generally favored in patients who prefer oral over intravenous/subcutaneous administration of medications and in those with preexisting neuropathy. In this regard, triplet all-oral combination of oral proteasome inhibitor ixazomib/Rd represents a valuable addition, considering its safety, tolerability, and activity in a Phase I/II trial in relapsed/refractory MM patients. ${ }^{30}$ Ixazomib/Rd could be a convenient and effective regimen in the near future if ongoing randomized studies that are investigating weekly dosing of ixazomib $4.0 \mathrm{mg}$ (versus placebo) plus Rd in relapsed/ refractory MM patients (NLM identifier: NCT01564537) and in previously untreated transplant-ineligible MM patients (NLM identifier: NCT01850524) substantiate its superiority. In addition, financial considerations become important in the choice of long-term therapy with lenalidomide-based regimens. In many countries, there is prohibitive out-ofpocket cost for using lenalidomide, and Rd given for many years may not be a financially viable option, even though it is a well-tolerated oral regimen ideal for elderly patients. The starting initial recommended dose of lenalidomide is $25 \mathrm{mg}$ daily for adults over 18 years of age. For patients above the age of 65 , dose reduction to $15 \mathrm{mg}$ or $10 \mathrm{mg}$ daily may be appropriate. ${ }^{31}$ Nearly all trials have shown that lenalidomide therapy increases the risk of venous thromboembolism (VTE), and the risk significantly increases when lenalidomide is combined with dexamethasone, melphalan, and prednisone. Aspirin, warfarin, and enoxaparin have all been used at various doses as VTE prophylaxis in trials of thalidomide and lenalidomide. In the two key randomized studies comparing RD to high-dose dexamethasone, VTE frequency of about $14 \%$ was noted with RD compared with a frequency of $5 \%$ in the dexamethasone group..$^{20,21}$ The exact mechanism by which lenalidomide and other IMiDs induce thrombosis is unknown, but it is thought that alterations in thrombomodulin, protease activated receptor- 1 , and resistance to activated protein $\mathrm{C}$ might play roles. ${ }^{32,33}$ Patients receiving combination therapy with lenalidomide should receive some form of thromboprophylaxis incorporated into their therapy, and modifiable risk factors should be managed. The most common hematologic toxicities associated with lenalidomide are grade 3/4 neutropenia and thrombocytopenia. Rates of these toxicities varied among trials and may have been affected by the context in which lenalidomide was used (ie, relapsed or refractory disease versus newly diagnosed $\mathrm{MM}$ ), but neutropenia occurred in $50 \%-60 \%$ of patients, and thrombocytopenia occurred in $20 \%-30 \%$ of patients. Patients should be closely monitored for hematologic toxicities and recommended dosage adjustments for lenalidomide should 
be followed with occurrence of grade 3 or 4 toxicities. Other common toxicities include fatigue, asthenia, nausea, constipation, muscle cramp, anemia, diarrhea, and rash. Fatigue is commonly associated with cumulative effect; although not generally severe, it can be bothersome for some patients, and can be minimized by supportive measures including moderate level of activity, proper fluid intake, and consistent sleeping schedule. In the lenalidomide maintenance studies, around $12 \%-27 \%$ of patients needed to discontinue therapy due to adverse events. ${ }^{27-29}$ Patients who discontinue for reasons other than progressive disease do poorly and hence optimal management of toxicities and dose modification strategies are crucial. Lenalidomide is mainly eliminated unchanged via the kidneys, and renal impairment reduces clearance of lenalidomide. As a result, lenalidomide dose adjustments are needed in patients with renal impairment to avoid excessive and potentially toxic levels of the drug. Lenalidomide is associated with risk of teratogenesis. Pregnancy must be ruled out before starting treatment in women of child-bearing age, and these women must use effective contraception while on lenalidomide. Lenalidomide is approved for marketing in the United States only under a FDA-approved restricted distribution program called Revlimid risk evaluation and mitigation strategy (REMS). As a requirement of the REMS program, access to this medication is restricted. Prescribers and pharmacies must be certified with the program to prescribe or dispense lenalidomide and patients must comply with the program requirements. Lenalidomide has been linked to second primary malignancies in MM. In trials of lenalidomide maintenance, there was a $7 \%-8 \%$ rate of second primary cancers in the lenalidomide groups compared with $3 \%-4 \%$ in the placebo groups. ${ }^{27-29}$ This increase in second cancers led to discontinuation of lenalidomide in the trial reported by Attal et al. ${ }^{27}$ In a meta-analysis including seven trials, the 5-year cumulative incidence of secondary primary hematologic malignancies was higher in patients treated with lenalidomide than in those who were not $(3.1 \%$ versus $1.4 \%$; HR $3.8 ; P=0.029) .{ }^{34}$ In addition, the risk was significantly higher in patients treated with lenalidomide plus oral melphalan than in those treated with melphalan alone (HR 4.86; $P<0.0001) .{ }^{34}$ The second primary cancers may be attributed to other factors, some of which include prior use of chemotherapeutic agents with known leukemogenic potential, MM-related disease factors, host-related factors, as well as environmental and behavioral factors. ${ }^{35}$

In summary, over the past decade, lenalidomide's use across multiple areas of the treatment paradigm has contributed greatly to improvements in the outcomes for MM.
Advances in our understanding of the molecular mechanisms of lenalidomide combined with an expanding repertoire of therapeutic options including monoclonal antibodies will herald effective combinations and further extend survival in MM patients.

\section{MDS}

Lenalidomide was approved by the FDA in 2005 for patients with transfusion-dependent anemia resulting from International Prognostic Scoring System (IPSS) low- or intermediate-1-risk MDS associated with the deletion $5 \mathrm{q}$ cytogenetic abnormality in the presence or absence of other cytogenetic abnormalities. Deletions of $5 \mathrm{q}$ occur in approximately $12 \%$ of MDS patients and represent the most common karyotypic abnormality in MDS. ${ }^{36}$ The " 5 q minus syndrome", accounting for $<5 \%$ of MDS cases and occurring mostly in older females, is characterized by an isolated $5 q$ deletion, macrocytic anemia, the absence of neutropenia, and preserved or increased platelet counts. ${ }^{37}$

Since many MDS patients are red cell transfusion dependent, need exists for drugs that can increase the hemoglobin in this population. Lenalidomide can reduce transfusion requirements in MDS patients. A Phase II study of 148 red blood cell transfusion-dependent patients evaluated the safety and efficacy of lenalidomide in IPSS low- or intermediate-1-risk MDS characterized by the $5 \mathrm{q} 31$ deletion. ${ }^{38}$ Patients received $10 \mathrm{mg}$ of lenalidomide daily or $10 \mathrm{mg}$ on days 1-21 of a 28 day cycle. Following 24 weeks of therapy, 112 patients (76\%) exhibited a reduction in the need for transfusions and 99 patients $(67 \%)$ had become transfusion-independent. Median time to response was 4.6 weeks, and median duration of response was 44 weeks. Neutropenia and thrombocytopenia were the most common adverse effects. Grade 3 or 4 neutropenia was seen in $55 \%$ of patients, and grade 3 or 4 thrombocytopenia was observed in $44 \%$ of patients.

Subsequently, a Phase III study examined the safety and efficacy of lenalidomide in 205 red blood cell transfusiondependent patients with IPSS low- or intermediate-1-risk MDS characterized by the $5 \mathrm{q} 31$ deletion. ${ }^{39}$ Patients were randomized to one of three arms: lenalidomide $10 \mathrm{mg}$ on days 1-21 of a 28 day cycle, lenalidomide $5 \mathrm{mg}$ daily, or placebo. Patients on the lenalidomide arms were significantly more likely to achieve transfusion independence compared to placebo. Transfusion independence, in turn, was associated with reductions in the risk of death and the risk of progression to acute myeloid leukemia (AML).

Although it is now accepted that lenalidomide has higher response rates in MDS patients with the $5 \mathrm{q}$ deletion 
than in those without this abnormality, this agent does have efficacy in patients whose disease lacks this abnormality. In a Phase I/II study, List et al examined 43 MDS patients with transfusion-dependent or symptomatic anemia who either had no response to erythropoietin stimulating agents or had an endogenous erythropoietin level $>500 \mathrm{mU} / \mathrm{mL} .{ }^{40}$ Patients received lenalidomide at doses of $25 \mathrm{mg}$ or $10 \mathrm{mg}$ daily or $10 \mathrm{mg}$ on days $1-21$ of a 28 day cycle. Overall, $56 \%$ of patients exhibited a sustained improvement in their hemoglobin. In patients with $5 \mathrm{q} 31$ deletions, the response rate was $83 \%$, compared to response rates of $57 \%$ and $12 \%$, respectively, for patients with normal karyotypes and other cytogenetic abnormalities. Additionally, in a Phase II trial, lenalidomide was examined in patients with transfusiondependent IPSS- low- and intermediate-1-risk MDS with karyotypes other than deletion $5 \mathrm{q} .{ }^{41}$ In this study, 214 patients received lenalidomide at a dose of $10 \mathrm{mg}$ daily or $10 \mathrm{mg}$ on days $1-21$ of a 28 day cycle. Twenty-six percent of patients achieved transfusion independence. Grade 3 or 4 neutropenia was observed in $30 \%$ of patients, and grade 3 or 4 thrombocytopenia was seen in $25 \%$ of patients.

In a retrospective study that utilized data gathered from two Phase II trials described above, ${ }^{38,41}$ Sekeres et al investigated the relationship of treatment-related cytopenias and response to lenalidomide in patients with lower-risk MDS. ${ }^{42}$ In these studies, MDS patients with the 5q deletion developed treatment-related thrombocytopenia at higher rates than patients without the $5 \mathrm{q}$ abnormality. Among patients with the $5 \mathrm{q}$ deletion, $70 \%$ percent of patients whose platelet count decreased by $50 \%$ or more experienced transfusion independence, compared to transfusion independence in only $42 \%$ in patients whose platelet counts decreased by less than 50\%. Moreover, among patients with the $5 \mathrm{q}$ deletion who did not have baseline neutropenia, $82 \%$ of those whose absolute neutrophil count decreased by $75 \%$ or more achieved transfusion independence, compared with $51 \%$ of those whose absolute neutrophil count remained stable. The authors conclude that their results corroborate a direct cytotoxic effect specific to the clone possessing the $5 \mathrm{q}$ deletion.

Lenalidomide is not currently approved for IPSS intermediate-2- or high-risk MDS but has been used in this setting. In a Phase II trial, 47 patients with higher-risk MDS harboring the $5 \mathrm{q}$ deletion received lenalidomide at a dose of $10 \mathrm{mg}$ daily. Twenty-seven percent of patients achieved a hematologic response, including seven patients who achieved morphologic complete remission (CR). Interestingly, 35\% of patients with initial platelet counts greater than $100,000 / \mathrm{mm}^{3}$ achieved CR, compared to none of the patients with platelet counts lower than $100,000 / \mathrm{mm}^{3}$.

\section{AML}

Lenalidomide is not approved for use in AML but has been used increasingly in the setting of relapsed/refractory AML and in older patients with AML. One report describes sustained morphologic and cytogenetic CR in two AML patients, ages 71 and 68 years, treated with high-dose single-agent lenalidomide. ${ }^{43}$ Each patient had trisomy 13 as their sole cytogenetic abnormality. Moreover, high-dose lenalidomide has been examined in a Phase II study of patients 60 years of age or older. ${ }^{44}$ Patients received up to two 28 -day cycles of lenalidomide at a dose of $50 \mathrm{mg}$ daily followed by lowdose maintenance (10 mg daily). Thirty percent of patients achieved CR or CR with incomplete recovery of blood counts (CRi). CR/CRi was seen only in patients who had white blood cell counts $<10,000 / \mu \mathrm{L}$ and peripheral blood blast counts $<1,000 / \mu \mathrm{L}$. In the group of patients who achieved $\mathrm{CR} / \mathrm{CRi}$, median duration of remission was 10 months.

Another Phase II study examined lenalidomide in previously untreated older AML patients (age $>60$ years) with deletion 5q. ${ }^{45}$ Patients received lenalidomide $50 \mathrm{mg}$ daily for 28 days, followed by a maintenance dose of $10 \mathrm{mg}$ on days 1-21 of a 28 day cycle. Of 37 evaluable patients, five (14\%) achieved a partial response (PR) or CR, two of whom had isolated deletion $5 \mathrm{q}$ and the other three of whom had complex cytogenetics. In addition, Chen et al studied single-agent lenalidomide in $18 \mathrm{relapsed} /$ refractory AML patients and nine high-risk MDS patients with the $5 \mathrm{q}$ deletion. ${ }^{46}$ Most patients had complex cytogenetics. Two AML patients (11\%) achieved CR or CR without platelet recovery. Interestingly, both of these patients had a trisomy 8 cytogenetic abnormality in a clone separate from the $5 \mathrm{q}$ deletion clone. Of note, no responses were observed in patients with complex cytogenetics.

Given the comparatively modest efficacy of lenalidomide in higher-risk MDS and AML, this agent is being explored in combinations with other agents. Recently, investigators have postulated that azacitidine and lenalidomide may have synergistic effects, in part because P53 mutations are associated with lenalidomide treatment failure and because azacitidine has activity in P53-mutated MDS. ${ }^{47}$ In addition, it has been noted that the effects of azacitidine are dependent on the cell cycle; since lenalidomide inhibits cell cycle progression, it has been suggested that sequential administration of azacitidine followed by lenalidomide is a rational approach. ${ }^{47}$ 
Multiple studies have tested the combination of azacitidine and lenalidomide in higher-risk MDS and AML, and various schedules of administration have been investigated. ${ }^{48-54}$ Lenalidomide was combined with azacitidine in a Phase I study of patients with higher-risk MDS or AML. ${ }^{48}$ Twenty patients with the $5 \mathrm{q}$ deletion (most with complex cytogenetics) were enrolled, seven of whom had a diagnosis of AML. Patients received azacitidine $75 \mathrm{mg} / \mathrm{m}^{2} /$ day subcutaneously on days $1-5$ and then were treated with lenalidomide on days 6-19 at escalating doses beginning at $10 \mathrm{mg}$ daily. Patients received up to eight cycles of this therapy. Individuals achieving complete clearance of bone marrow blasts following two or more cycles of treatment then received maintenance azacitidine $30 \mathrm{mg} / \mathrm{m}^{2} /$ day and lenalidomide at the maximum tolerated dose on days 6-19. Overall, $26 \%$ of patients achieved a hematologic response (defined as $\mathrm{CR}, \mathrm{CRi}$, or PR), and $42 \%$ exhibited a cytogenetic response. Among patients who were previously untreated, hematologic and cytogenetic response rates were $44 \%$ and $56 \%$. In another small study, 18 AML patients ages 60 years and older, most of whom did not have the $5 \mathrm{q}$ deletion, were treated with sequential azacitidine and lenalidomide. Forty-four percent of evaluable patients achieved CR or CRi. ${ }^{50}$ Importantly, the successful use of azacitidine and lenalidomide together has been reported as a bridge to allogeneic hematopoietic cell transplantation in relapsed/refractory AML patients. ${ }^{48,52,55}$

Other lenalidomide combinations are under exploration. Lenalidomide and bortezomib have been used together, with some MDS and AML patients achieving CR. ${ }^{56}$ Lenalidomide has also been tested together with cytarabine, daunorubicin, and etoposide in higher-risk MDS and AML with the $5 \mathrm{q}$ deletion; a subset of patients achieved CR. ${ }^{57}$

In summary, IPSS low- and intermediate-1 risk MDS patients can be treated with supportive care, lenalidomide, or hypomethylating agents. Patients with lower-risk MDS characterized by the $5 \mathrm{q}$ deletion are more likely to benefit from lenalidomide. Lenalidomide also has some activity in MDS patients lacking the $5 \mathrm{q}$ abnormality. This agent can often be useful in those lower-risk patients who are refractory to erythropoietin stimulating agents or have high baseline erythropoietin levels. Higher-risk MDS and AML (especially relapsed/refractory AML and elderly AML) present significant treatment challenges, including the limited number of efficacious treatment options. Lenalidomide and lenalidomide-containing combinations have shown some activity in these diseases, and lenalidomide has now become part of the arsenal to treat these illnesses. Sequential azacitidine and lenalidomide has some efficacy and may be an appropriate choice in carefully selected relapsed/refractory AML patients, and also as initial therapy in some older AML patients.

\section{Lymphoma}

Lenalidomide has shown clinical activity in non-Hodgkin and Hodgkin lymphoma. Table 2 summarizes the key clinical studies in mantle cell lymphoma (MCL), diffuse large B cell lymphoma (DLBCL), and indolent lymphoma. Table 3 summarizes the published clinical trials in chronic lymphocytic leukemia.

\section{MCL}

Lenalidomide has antitumor activity in MCL as a single agent and in combination with rituximab. The earliest report of single-agent lenalidomide in MCL was a subset analysis by Haberman et al in 15 patients with relapsed/refractory MCL. ${ }^{58}$ The overall response rate was $53 \%$ and $\mathrm{CR}$ rate was $20 \%$. The median PFS was 5.6 months. A larger study which included 57 patients with relapsed/refractory MCL demonstrated ORR and CR rate of $35 \%$ and $12 \%$, respectively, and the median PFS was 8.8 months. ${ }^{59}$ In both studies, lenalidomide was used as a single agent at a dose of $25 \mathrm{mg}$ daily for days 1-21 of a 28 day cycle. The studies included patients with relapsed refractory aggressive lymphoma including MCL. Eve et al reported a regimen of lenalidomide $25 \mathrm{mg}$ per day on days 1-21 followed by lenalidomide $15 \mathrm{mg}$ per day on days $1-21$ of a 28 day schedule until progression for responding patients. ${ }^{60}$ The ORR was $31 \%$ and $C R$ rate was $8 \%$. Patients who did not receive maintenance lenalidomide had a PFS of 3.9 months compared to 14.6 months in patients who had maintenance. It should be noted that only responding patients received maintenance lenalidomide which could have accounted for the difference in PFS between the two groups.

The EMERGE trial tested the use of single-agent lenalidomide for patients with MCL who relapsed on or were refractory to bortezomib. ${ }^{61}$ In this study, an ORR of $28 \%$ and CR rate of $8 \%$ were reported. The median time to response was 2.2 months, median duration of response was 16.6 months, and median PFS and OS were 4 and 19 months, respectively. The most common $\geq$ grade 3 adverse events were cytopenias. The results of this study led to the FDA approval of lenalidomide in patients with MCL whose disease had relapsed or progressed after two prior therapies, one of which included bortezomib.

The promising single-agent activity and the preclinical evidence that lenalidomide can potentiate the antibody dependent cellular cytotoxicity of rituximab ${ }^{62}$ led to studies 
Table 2 Key clinical trials in lymphoma with lenalidomide

\begin{tabular}{|c|c|c|c|c|c|c|}
\hline Study/author & Regimen & $\mathbf{N}$ & ORR \% & CR \% & PFS/TTP (m) & OS (m) \\
\hline \multicolumn{7}{|c|}{ Relapsed/refractory mantle cell lymphoma } \\
\hline Habermann et $\mathrm{a}^{58}$ & Len & 15 & 53 & 20 & 5.6 & NA \\
\hline \multirow[t]{2}{*}{ Eve et $\mathrm{al}^{60}$} & Len + /- Len maint & 26 & 31 & 8 & 3.9 no maint & Not reached \\
\hline & & & & & I4.6 with maint & \\
\hline Zinzani et a ${ }^{59}$ & Len & 57 & 35 & 12 & 8.8 & NA \\
\hline Goy et $\mathrm{al}^{61}$ & Len & 134 & 28 & 8 & 4 & 19 \\
\hline Wang et $\mathrm{a}^{63}$ & Len + rituximab & 52 & 57 & 36 & II.I & 24.3 \\
\hline Zaja et al ${ }^{64}$ & Len + Dex & 33 & 52 & 24 & 12 & 20 \\
\hline Morrison et a $\mathrm{a}^{66}$ & Len + bortezomib & 53 & 40 & 15 & I yr PFS $41 \%$ & I yr OS $67 \%$ \\
\hline \multirow[t]{2}{*}{ Trneny et al ${ }^{67}$} & Len & 167 & 40 & 5 & 8.7 & 27.8 \\
\hline & $I C^{\Omega}$ & 83 & 11 & 0 & 5.2 & 21.2 \\
\hline \multicolumn{7}{|c|}{ Newly diagnosed mantle cell lymphoma } \\
\hline Ruan et al ${ }^{68}$ & Len + rituximab & 38 & 89 & 58 & 2 yr PFS $84.6 \%$ & 2 yr OS $94.2 \%$ \\
\hline \multicolumn{7}{|c|}{ Relapsed/refractory diffuse large B cell lymphoma } \\
\hline Wiernik et al ${ }^{69}$ & Len & 26 & 19 & 12 & $4^{*}$ & $\mathrm{~N} / \mathrm{A}$ \\
\hline Witzig et $\mathrm{al}^{70}$ & Len & 108 & 28 & 7 & 2.7 & N/A \\
\hline Zinzani et a ${ }^{74}$ & Len + rituximab $\rightarrow$ Len & 23 & 35 & 35 & I yr DFS $34.8 \%$ & 18 mo OS $55 \%$ \\
\hline Wang et $\mathrm{al}^{75}$ & Len + rituximab & 32 & 28 & 22 & 2.8 & 10.2 \\
\hline Feldman et $\mathrm{al}^{76}$ & Len + RICE ${ }^{*}$ & 15 & 73 & 60 & NA & NA \\
\hline \multirow{2}{*}{ Czuczman et al ${ }^{17}$} & Len & 51 & 28 & 0 & I3.6 weeks & 31 \\
\hline & $\mathrm{IC}^{ \pm}$ & 51 & 12 & 0 & 7.9 weeks & 24.6 \\
\hline \multicolumn{7}{|c|}{ Newly diagnosed diffuse large B cell lymphoma } \\
\hline Chiappella et $\mathrm{al}^{78}$ & Len $+\mathrm{RCHOP}$ & 21 & 90 & 81 & NA & NA \\
\hline Vitolo et $\mathrm{al}^{79}$ & Len + RCHOP & 49 & 92 & 86 & 2 yr PFS $80 \%$ & 2 yr OS $92 \%$ \\
\hline Nowakowski et al ${ }^{80}$ & Len + RCHOP & 64 & 98 & 80 & 2 yr EFS $59 \%$ & 2 yr OS $78 \%$ \\
\hline \multicolumn{7}{|c|}{ Relapsed/refractory indolent lymphoma } \\
\hline Witzig et al $7^{70}$ & Len & 43 & 23 & 7 & 4.4 & NA \\
\hline Tuscano 82 & Len + rituximab & 27 & 74 & 44 & 12.4 & NA \\
\hline \multicolumn{7}{|c|}{ Newly diagnosed indolent lymphoma } \\
\hline \multirow[t]{2}{*}{ Kimby et a ${ }^{85}$} & Len + rituximab & 77 & 87 & 42 & NA & NA \\
\hline & Rituximab & 77 & 66 & 28 & NA & NA \\
\hline Fowler et a ${ }^{84}$ & Len + rituximab & 110 & 90 & 65 & 53.8 & 3 yr OS $96.1 \%$ \\
\hline
\end{tabular}

Notes: *Value for whole study group; ${ }^{*}$ eligible patients were allowed to proceed to an autologous stem cell transplant followed by lenalidomide maintenance for I year; IIC included single-agent gemcitabine, oxaliplatin, rituximab, or etoposide; ${ }^{\Omega} \mathrm{C}$ included single-agent gemcitabine, cytarabine, or fludarabine.

Abbreviations: CR, complete response; Dex, dexamethasone; DFS, disease free survival; EFS, event free survival; Len, lenalidomide; maint, maintenance; ORR, overall response rate; OS, overall survival; PFS, progression-free survival; RCHOP, rituximab, cyclophosphamide, doxorubicin, vincristine, and prednisone; RICE, rituximab, ifosfamide, carboplatin, and etoposide; TTP, time to progression; NA, not available; IC, investigator choice.

using the combination of lenalidomide with rituximab. Wang et al reported this combination in 52 patients with relapsed/refractory MCL; the ORR of 57\%, CR 36\%, PFS 11.1 months, respectively, were clearly superior compared to single-agent studies with lenalidomide. ${ }^{63}$
Zaja et al reported a 52\% ORR, PFS of 12 months, and median OS of 20 months in 33 patients with the combination of lenalidomide and dexamethasone. ${ }^{64} \mathrm{~A}$ randomized comparison of the combination (lenalidomide plus rituximab) versus single-agent lenalidomide has not been reported. The combi-

Table 3 Key clinical trials in CLL with lenalidomide

\begin{tabular}{|c|c|c|c|c|c|c|c|}
\hline Study/author & Regimen & $\mathbf{N}$ & ORR \% & CR \% & PFS/TTP (m) & OS (m) & Median FU (m) \\
\hline \multicolumn{8}{|c|}{ Relapsed/refractory chronic lymphocytic leukemia } \\
\hline Chanan-Khan et $\mathrm{al}^{86}$ & Len & 45 & 47 & 9 & NA & NA & NA \\
\hline Ferrajoli et al ${ }^{87}$ & Len & 44 & 32 & 7 & NA & $73 \%$ & 14 \\
\hline Badoux et $a^{88}$ & Len + rituximab & 59 & 66 & 12 & Median TTF 17.4 & 3 yr OS $71 \%$ & 33 \\
\hline \multicolumn{8}{|c|}{ Newly diagnosed chronic lymphocytic leukemia } \\
\hline Badoux et al ${ }^{89}$ & Len & 60 & 65 & 10 & 2 yr PFS $60 \%$ & $88 \%$ & 29 \\
\hline Chen et $\mathrm{al}^{90}$ & Len & 25 & 56 & 0 & 2 yr PFS 89\% & 2 yr OS $92 \%$ & 20.7 \\
\hline James et $\mathrm{al}^{91}$ & Len + rituximab & 69 & 88 & 15 & 20 & $93 \%$ & 20 \\
\hline
\end{tabular}

Abbreviations: CLL, chronic lymphocytic leukemia; CR, complete response; FU, follow-up; Len, lenalidomide; ORR, overall response rate; OS, overall survival; PFS: progressionfree survival; TTP, time to progression; NA, not available; TTF, time to treatment failure. 
nation of lenalidomide and bortezomib has shown significant synergy in MM. Two trials have studied this combination in MCL. Flinn et al studied the combination of lenalidomide, bortezomib, and rituximab in a Phase I/II trial in 22 patients, 16 of whom had received no prior therapy and the remainder had received one prior therapy. ${ }^{65}$ Lenalidomide was dosed at $10 \mathrm{mg}$ daily on days $1-14$; bortezomib at $1.3 \mathrm{mg} / \mathrm{m}^{2}$ intravenously on days $1,4,8$, and 11; and rituximab at $375 \mathrm{mg}$ / $\mathrm{m}^{2}$ on days 1,8 , and 15 of cycle 1 and day 1 of subsequent cycles. The ORR, CR, and 18-month PFS for the entire group were $82 \%, 32 \%$, and $61 \%$, respectively. For patients who had not received prior therapy, the ORR was $75 \%$. Morrison et al reported an ORR of $40 \%$ and 1-year PFS of $41 \%$ in relapsed/refractory MCL. ${ }^{66}$ Unfortunately the incidence of grade 3 neuropathy was $32 \%$ which could have been due to the intravenous use of bortezomib.

Recently, Trneny et al reported a randomized comparison of lenalidomide versus investigator's choice (IC) (single-agent gemcitabine, cytarabine, or fludarabine).$^{67}$ Lenalidomide demonstrated a significant improvement in ORR (40\% versus $11 \%, P<0.001)$ and PFS (8.7 months versus 5.2 months, HR $0.61, P=0.004)$ over the IC arm. Duration of response was not statistically significant between the two groups: 16 months versus 10.4 months for lenalidomide and IC, respectively. Crossover to the lenalidomide arm was allowed and the difference in OS between the two arms (27.8 months versus 21.2 months) was not statistically significant.

In previously untreated MCL, the combination of lenalidomide and rituximab was reported recently by Ruan et al. Lenalidomide was used at $20 \mathrm{mg}$ (escalated to $25 \mathrm{mg}$ if tolerated) on days $1-21$ of a 28 day cycle for 12 cycles followed by $15 \mathrm{mg}$ as a maintenance dosing till progression. Rituximab was given weekly for 4 weeks followed by every 4 weeks for 2 years. ${ }^{68}$ Thirty-two percent of patients were at high risk by the Mantle Cell International Prognostic Index (MIPI) and 21\% had Ki67 >30\%. In the evaluable population, ORR, CR, and PR were $89 \%, 58 \%$, and $31 \%$, respectively. Twenty-four-month PFS and OS were $84.6 \%$ and $94.2 \%$, respectively. Neither MIPI score nor Ki67 correlated with response. Median time to objective response was 2.8 months and median time to CR was 11 months.

In summary, single-agent lenalidomide has modest activity in relapsed/refractory MCL, and achieves higher response rates in combination with rituximab. Combinations with bortezomib have been limited by the incidence of neuropathy; perhaps the use of subcutaneous bortezomib will be able to mitigate this effect. Recently, the BTK inhibitor ibrutinib has been FDA approved in relapsed/refractory MCL and combination studies with lenalidomide in non-Hodgkin lymphoma including MCL are being performed. The high response rate of lenalidomide and rituximab in previously untreated MCL is very encouraging, although the long-term durability of the response is currently unknown. In the frontline setting, there is a clinical trial exploring the combination of lenalidomide with rituximab-bendamustine and other studies looking at the role of lenalidomide maintenance after completion of a rituximab chemotherapy induction.

\section{DLBCL}

Two clinical trials, NHL-002 and NHL-003, showed that singleagent lenalidomide has clinical activity in DLBCL. In NHL002, the ORR and $\mathrm{CR} /$ complete remission unconfirmed $(\mathrm{CRu})$ rate were $19 \%$ and $12 \%$, respectively, in the DLBCL cohort which included 26 patients. ${ }^{69}$ This trial also included patients with follicular grade 3 lymphoma, MCL, and transformed indolent lymphoma. PFS and median duration of response for the DLBCL cohort were not reported, but for the entire group were 4 months and 6.2 months, respectively. The median time to $\mathrm{CR}$ was 4.3 months and median time to PR was 1.9 months. In the NHL-003 trial, ORR and CR rate were $28 \%$ and $7 \%$, respectively, in 108 patients with relapsed/refractory DLBCL. ${ }^{70}$ In this larger study, the median PFS and duration of response (DOR) were 2.7 months and 4.6 months, respectively.

DLBCL has been subclassified into germinal center type (GCB), ABC, and primary mediastinal subtype based on gene expression profiling. ${ }^{71}$ Patients with $\mathrm{ABC}$ subtype have a significantly worse outcome when treated with frontline chemotherapy and therefore account for more relapses than the other subtypes. ${ }^{72}$ Hernandez-Ilizaliturri analyzed the activity of lenalidomide in relapsed/refractory DLBCL in GCB-like and non-GCB-like subtypes according to the immunohistochemistry based Hans algorithm. In this retrospective study, single-agent lenalidomide had a significant preferential benefit in non-GCB-like as compared to GCB-like DLBCL (ORR 52.9\% versus 8.7\%). ${ }^{73}$

Two clinical studies have tested the combination of lenalidomide and rituximab in DLBCL. Zinzani et al reported this combination in elderly patients (aged $\geq 65$ years) with relapsed/refractory DLBCL. ${ }^{74}$ Lenalidomide was used at $20 \mathrm{mg}$ daily on days 1-21 of a 28 day cycle and rituximab was used on days 1 and 21. After 4 cycles, patients achieving stable disease or better received lenalidomide monotherapy as maintenance for 8 months. The $\mathrm{CR}$ rate at the end of maintenance, 12 month disease-free survival, and 18 month OS were $35 \%, 35 \%$, and $55 \%$, respectively. The second study was reported by Wang et al in 32 patients with relapsed and refractory DLBCL. ${ }^{75}$ In this study, rituximab was used weekly for 4 weeks during cycle 1 . The ORR, CR rate, 
median PFS, and median OS were 28\%, 22\%, 2.8 months, and 10.2 months, respectively.

In relapsed transplant-eligible DLBCL, lenalidomide has been combined with rituximab, ifosfamide, carboplatin, and etoposide (RICE) in a Phase I/II trial. ${ }^{76}$ In this study, 15 patients received dose-escalated lenalidomide given for days 1-7, combined with RICE given every 2 weeks. After 3 cycles, patients with chemosensitive disease underwent autologous stem cell transplant followed by lenalidomide maintenance for up to 12 months. After 2 cycles of lenalidomide plus RICE, the ORR was $73 \%$ and CR was $60 \%$.

The first randomized study of lenalidomide versus IC (monotherapy with either gemcitabine, rituximab, etoposide, or oxaliplatin) was recently reported by Czuczman et al. ${ }^{77}$ Patients with DLBCL who received two or more prior therapies, or were ineligible for stem cell transplantation or further combination chemotherapy, were enrolled. Twenty-nine patients crossed over to the lenalidomide arm after progression on the IC arm. For all patients, there was a statistically significant difference in PFS favoring lenalidomide versus IC (13.6 weeks versus 7.9 weeks, HR 0.64, $P=0.041$ ). There was improved ORR, PFS, and OS with lenalidomide versus IC in the non-GCB population as defined by immunohistochemistry (IHC) (29\% versus 12\%, 15.1 weeks versus 7.1 weeks, 32.3 weeks versus 20.4 weeks), and the difference appears to be more pronounced in the ABC population as defined by GEP (46\% versus 19\%, 82 weeks versus 6.2 weeks, 108.4 weeks versus 18.6 weeks). For the subgroup analysis, the results were not statistically significant with the exception of the difference in PFS in the non-GCB population (HR $0.5, P=0.02)$.

In newly diagnosed DLBCL, lenalidomide combined with rituximab, cyclophosphamide, doxorubicin, vincristine, and prednisone (R-CHOP) in a Phase I study achieved ORR of $90 \%$ and CR of $81 \% .{ }^{78}$ In a Phase II trial, $15 \mathrm{mg}$ lenalidomide was combined with R-CHOP and ORR and CR rate were $92 \%$ and $86 \%$, respectively. ${ }^{79}$ After a median follow up of 28 months, the 2-year PFS and OS were $80 \%$ and $92 \%$, respectively. Nowakowski et al studied the addition of lenalidomide to R-CHOP for frontline therapy of DLBCL. ${ }^{80}$ The ORR and CR rate were $98 \%$ and $80 \%$, respectively. Event-free survival and OS at 2 years were $59 \%$ and $78 \%$, respectively. The results were compared to 87 historical control patients from the Mayo Clinic lymphoma database who were treated with R-CHOP. Interestingly, the addition of lenalidomide appeared to mitigate the negative impact of non-GCB phenotype on patient outcome.
Lenalidomide maintenance at $25 \mathrm{mg}$ daily for 21 days of a 28 day cycle for 1 year was tested after R-CHOP chemotherapy in newly diagnosed DLBCL. The 2-year disease-free survival and OS for patients who received lenalidomide maintenance were $90 \%$ and $95.2 \%$, respectively. ${ }^{81}$

In summary, the addition of lenalidomide to R-CHOP has yielded higher response rates compared to historical controls in previously untreated DLBCL. A randomized trial of $\mathrm{R}-\mathrm{CHOP}$ with and without lenalidomide is being conducted to confirm the benefit. Lenalidomide appears to overcome the poor prognostic impact of non-GCB DLBCL in both newly diagnosed and relapsed DLBCL. In relapsed DLBCL, single-agent lenalidomide has clinical activity but the limitations include the low CR rate, slow onset of response, and lack of durability. To overcome these limitations, various combinations of lenalidomide with antibodies: rituximab, obinutuzumab; antibody drug conjugate: brentuximab; BTK inhibitor: ibrutinib; mTOR inhibitors: temsirolimus, everolimus; and chemotherapy: R-CHOP, R-EPOCH, gemcitabine are being tested in clinical trials.

\section{Indolent lymphoma}

The early single-agent trials of lenalidomide in lymphoma included patients with follicular lymphoma. In NHL-001, 43 patients with indolent NHL were enrolled including 22 patients with follicular lymphoma grade 1 and $2 .^{70}$ The ORR and CR rate were $27 \%$ and $9 \%$, respectively. The median PFS for indolent lymphoma was 4.4 months. Lenalidomide plus rituximab was tested in 30 patients with relapsed/refractory indolent lymphoma. ${ }^{82}$ The ORR, CR rate, and median PFS were 74\%, 44\%, and 12.4 months, respectively. In a randomized trial of lenalidomide versus lenalidomide plus rituximab in relapsed follicular lymphoma, the ORR, CR rate, and median event-free survival were $75 \%$, $32 \%$, and 2 years for lenalidomide and rituximab compared to $49 \%, 13 \%$, and 1.2 years for lenalidomide alone. ${ }^{83}$

Lenalidomide plus rituximab has demonstrated excellent clinical activity in newly diagnosed indolent lymphoma. In a Phase II trial, 110 indolent lymphoma patients received lenalidomide $10 \mathrm{mg}$ /day escalated to $20 \mathrm{mg}$ /day and rituximab was administered on day 1 of every cycle. The ORR and CR rate were $90 \%$ and $65 \%$, respectively. Complete responses in follicular lymphoma, marginal zone lymphoma, and small lymphocytic lymphoma were $87 \%, 67 \%$, and $23 \%$, respectively. ${ }^{84}$ In a randomized trial, 154 patients with follicular lymphoma grade 1-3a received either rituximab on day 1 of weeks $1-4$ and $12-15$ or the same schedule of rituximab plus 
lenalidomide administered at $15 \mathrm{mg}$ daily, starting 14 days before the first dose of rituximab and taken continuously until 14 days after the last dose of rituximab. ${ }^{85}$ The ORR and $\mathrm{CR} / \mathrm{CRu}$ rate of lenalidomide plus rituximab were $87 \%$ and $42 \%$, respectively, compared to $66 \%$ and $28 \%$, respectively, for patients who received rituximab alone. The follow-up for this trial is short, and survival data are not available.

In summary, lenalidomide has promising activity in indolent lymphoma. As expected, the response rates of lenalidomide and rituximab are very high in newly diagnosed follicular lymphoma and a randomized trial has shown statistically significant improvement in $\mathrm{CR} / \mathrm{CRu}$ compared to rituximab alone. In relapsed indolent lymphoma, single-agent lenalidomide has modest activity, but when combined with rituximab the responses are robust and more durable when compared to lenalidomide alone.

\section{Chronic lymphocytic leukemia}

The first clinical demonstration of the activity of lenalidomide in chronic lymphocytic leukemia (CLL) was a Phase II trial in 45 patients with relapsed/refractory CLL who received $25 \mathrm{mg}$ of lenalidomide on days $1-21$ of a 28 day cycle. ${ }^{86}$ As a result of tumor lysis syndrome in two patients, the protocol was amended to start lenalidomide at $5 \mathrm{mg}$ and escalated by $5 \mathrm{mg}$ every 1-2 weeks to a maximum of $25 \mathrm{mg}$. Patients who had progressive disease or stable disease had rituximab added to lenalidomide. Tumor flare reaction was seen in $58 \%$ of patients; $8 \%$ of patients had grade 3 or 4 tumor flare which was treated with either ibuprofen or morphine. The last 16 patients to enroll in this trial received prophylaxis with oral prednisone. Tumor flare reaction is a unique side effect of lenalidomide in this disease and consists of swelling of the lymph nodes, spleen, and/or liver with or without fever, erythema, maculopapular nonpruritic rash, bone pain, and increase in lymphocyte counts. The ORR and CR rate were $47 \%$ and $9 \%$, respectively. Of the three patients who exhibited disease progression, addition of rituximab led to a PR. In another Phase II trial, lenalidomide was started at $10 \mathrm{mg}$ daily and increased by $5 \mathrm{mg}$ every 28 days to a maximum of $25 \mathrm{mg} .{ }^{87}$ The ORR and CR rate in 44 patients with relapsed/ refractory CLL were $32 \%$ and $7 \%$, respectively. In this study, the efficacy of lenalidomide was seen across high-risk groups such as del 17p, del 11q, and unmutated IGVH.

Badoux et al reported the combination of lenalidomide and rituximab in relapsed or refractory CLL. ${ }^{88}$ The ORR, CR rate, and 3 -year OS were $66 \%, 12 \%$, and $71 \%$, respectively. Time to treatment failure was 17.4 months. In this trial, rituximab was given weekly during cycle 1 and on day 1 of cycles 3-12. Lenalidomide was started on day 9 of cycle 1 at $10 \mathrm{mg}$ and administered continuously.

In newly diagnosed elderly (aged $\geq 65$ years) CLL, lenalidomide monotherapy yielded an ORR, CR rate, and 2-year PFS of $65 \%, 10 \%$, and $60 \%$, respectively. ${ }^{89}$ Chen et al reported results of a single-agent lenalidomide study in untreated CLL. ${ }^{90}$ The first two patients were started with $10 \mathrm{mg}$ lenalidomide for 21 days of a 28 day cycle and weekly escalations of $5 \mathrm{mg}$ dose to a maximum of $25 \mathrm{mg}$. Severe toxicities with tumor lysis and fatal sepsis were noted. The protocol was amended to start with $2.5 \mathrm{mg}$ of lenalidomide; with this dose, no further tumor lysis was noted. However, tumor flare was seen in $88 \%$ of patients and a high incidence of $\geq$ grade 3 neutropenia was observed in $72 \%$ of patients. The ORR was $56 \%$ and no CRs were seen. A large randomized study of lenalidomide versus chlorambucil called the ORIGIN trial was stopped early due to increased deaths in the lenalidomide arm compared to the chlorambucil arm. James et al studied the combination of lenalidomide and rituximab in previously untreated CLL. ${ }^{91}$ Sixty-nine patients were enrolled onto one of two age-specific strata. The median ages were 56 and 70 years for the two arms. The ORR and CR rate for the younger group were $95 \%$ and $20 \%$, respectively, and for the older group were $78 \%$ and $11 \%$, respectively. The median PFS did not differ between the younger and older cohort: 19 and 20 months, respectively.

To summarize, in previously untreated CLL, lenalidomide has significant activity but clinical development has been impaired due to significant toxicity. The use of very low doses of lenalidomide and vigilance for tumor lysis syndrome is warranted. In relapsed and refractory CLL, the combination of rituximab and lenalidomide leads to higher and more durable responses than lenalidomide alone, although a randomized comparison has not been reported. The adverse effects of tumor lysis and tumor flare appear to be unique to lenalidomide and CLL. It is important to recognize tumor flare and avoid confusion with disease progression.

\section{T cell lymphoma}

Zinzani et al reported the first study to demonstrate activity of lenalidomide in T-cell lymphoma. ${ }^{92}$ In this Phase II trial, lenalidomide was used at a dose of $25 \mathrm{mg}$ in ten patients with relapsed and refractory PTCL-nos. The ORR and CR rate was $30 \%$ (3/10 patients). However, two of the three patients who achieved a CR relapsed less than 5 months after completing treatment. The EXPECT trial was a larger trial of single-agent 
lenalidomide in 54 patients with relapsed/refractory PTCL. ${ }^{93}$ The ORR and CR/CRu rate were $22 \%$ and $11 \%$, respectively. The median PFS and DOR were short at 2.5 months and 3.6 months, respectively. Lenalidomide has been combined with vorinostat in a Phase I trial. ${ }^{94}$ Due to toxicity, the MTD of lenalidomide on this trial was $5 \mathrm{mg}$ and only two of eight patients responded to the combination.

\section{Classical Hodgkin lymphoma}

Lenalidomide has modest clinical activity in patients with relapsed and refractory Hodgkin lymphoma. In a Phase II trial of 38 heavily pretreated patients with classical Hodgkin lymphoma, ORR rate was $19 \%$ and one patient achieved a $\mathrm{CR} .{ }^{95}$ Median duration of response was 6 months and median time to treatment failure was 15 months. Combinations of lenalidomide with chemotherapy and/or antibody drug conjugates such as brentuximab have not been reported in classical Hodgkin lymphoma.

\section{Impact on quality of life}

Although lenalidomide is a fairly well-tolerated drug, its use is associated with side effects such as cytopenias, rash, gastrointestinal symptoms, infections, and venous thromboembolic events. Therefore, an important consideration is the impact of this drug on the quality of life. In del 5q MDS, the use of lenalidomide is associated with a reduced requirement for transfusions. In a randomized trial of lenalidomide versus placebo in patients with IPSS low-/intermediate-1-risk del $5 q$ MDS, health-related quality of life (HRQoL) outcomes were assessed using the Functional Assessment of Cancer Therapy-Anemia (FACT-An). ${ }^{96}$ Mean baseline to 12 -week changes in FACT-An total scores improved following treatment with lenalidomide $(+5.7)$ versus placebo $(-2.8)$. Longterm improvements at 48 weeks were also seen. The authors concluded that in del 5q MDS, the improvements in red blood cell transfusion independence and hemoglobin level are associated with patient functioning and well-being.

In MM, the MM-015 trial assessed the effect of lenalidomide-based therapy (melphalan-prednisone-lenalidomide) followed by lenalidomide maintenance on HRQoL. Patients completed HRQoL questionnaires at baseline, after every 3rd treatment cycle, and at the end of treatment. Patients receiving lenalidomide maintenance exhibited pronounced improvements in quality of life including physical functioning. ${ }^{97}$ In the FIRST trial, continuous lenalidomide and dexamethasone treatment improved HRQoL measurements compared to MPT. ${ }^{98}$ This improvement was mainly associated with improvements in PFS.
These studies indicate that the clinical benefits from lenalidomide lead to improvement in quality of life and may outweigh some of the side effects of the drug.

\section{Conclusion}

Lenalidomide is an IMiD that has effects on tumor cells and the tumor microenvironment leading to clinical efficacy across a diverse spectrum of hematologic malignancies. Recent insights into the biology of IMiDs have identified cereblon as a crucial molecule for the lenalidomide effect and may lead to development of biomarkers for sensitivity or resistance to lenalidomide. Lenalidomide is currently FDA approved for the use: 1) in patients with transfusiondependent anemia due to low- or intermediate-1-risk MDS associated with del 5q; 2) in combination with dexamethasone in patients with $\mathrm{MM}$ who have received one prior therapy; and 3) in patients with MCL whose disease has relapsed or progressed after two prior therapies, one of which included bortezomib. In addition to the above indications, we have reviewed the clinical efficacy in newly diagnosed myeloma, MDS/AML, NHL, CLL, and Hodgkin lymphoma. Lenalidomide has the advantages of oral administration and manageable toxicity. Combinations of lenalidomide with traditional chemotherapy and novel biologic agents are being explored in clinical trials. Novel agent combinations have become standard of care in MM therapy and the progress in other hematologic malignancies is promising.

\section{Disclosure}

NG: Advisory Board member and Speakers Bureau: Celgene. MRG, OF, and MB report no conflicts of interest in this work.

\section{References}

1. Henry JY, Labarthe MC, Meyer B, Dasgupta P, Dalgleish AG, Galustian C. Enhanced cross-priming of naive CD8+ T cells by dendritic cells treated by the $\mathrm{IMiDs}^{\circledR}$ immunomodulatory compounds lenalidomide and pomalidomide. Immunology. 2013;139:377-385.

2. Castelli R, Cassin R, Cannavò A, Cugno M. Immunomodulatory drugs: new options for the treatment of myelodysplastic syndromes. Clin Lymphoma Myeloma Leuk. 2013;13:1-7.

3. LeBlanc R, Hideshima T, Catley LP, et al. Immunomodulatory drug costimulates T cells via the B7-CD28 pathway. Blood. 2004;103: $1787-1790$.

4. Dredge K, Marriott JB, Macdonald CD, et al. Novel thalidomide analogues display anti-angiogenic activity independently of immunomodulatory effects. Br J Cancer. 2002;87:1166-1172.

5. Martiniani R, Di Loreto V, Di Sano C, Lombardo A, Liberati AM. Biological activity of lenalidomide and its underlying therapeutic effects in multiple myeloma. Adv Hematol. 2012;2012:842945.

6. Mitsiades CS, Mitsiades NS, Richardson PG, Munshi NC, Anderson KC. Multiple myeloma: a prototypic disease model for the characterization and therapeutic targeting of interactions between tumor cells and their local microenvironment. J Cell Biochem. 2007;101:950-968. 
7. Verhelle D, Corral LG, Wong K, et al. Lenalidomide and CC-4047 inhibit the proliferation of malignant $\mathrm{B}$ cells while expanding normal CD34+ progenitor cells. Cancer Res. 2007;67:746-755.

8. Escoubet-Lozach L, Lin IL, Jensen-Pergakes K, et al. Pomalidomide and lenalidomide induce $\mathrm{p} 21 \mathrm{WAF}-1$ expression in both lymphoma and multiple myeloma through a LSD1-mediated epigenetic mechanism. Cancer Res. 2009;69:7347-7356.

9. Ito $\mathrm{T}$, Ando $\mathrm{H}$, Suzuki T, et al. Identification of a primary target of thalidomide teratogenicity. Science. 2010;327:1345-1350.

10. Lopez-Girona A, Mendy D, Ito T, et al. Cereblon is a direct protein target for immunomodulatory and antiproliferative activities of lenalidomide and pomalidomide. Leukemia. 2012;26:2326-2335.

11. Zhang LH, Kosek J, Wang M, Heise C, Schafer PH, Chopra R. Lenalidomide efficacy in activated B-cell-like subtype diffuse large B-cell lymphoma is dependent upon IRF4 and cereblon expression. Br J Haematol. 2013;160:487-502.

12. Zhu YX, Braggio E, Shi CX, et al. Cereblon expression is required for the antimyeloma activity of lenalidomide and pomalidomide. Blood 2011;118:4771-4779.

13. Heintel D, Rocci A, Ludwig H, et al. High expression of cereblon (CRBN) is associated with improved clinical response in patients with multiple myeloma treated with lenalidomide and dexamethasone. $\mathrm{Br} J$ Haematol. 2013;161:695-700.

14. Lu G, Middleton RE, Sun H, et al. The myeloma drug lenalidomide promotes the cereblon-dependent destruction of Ikaros proteins. Science. 2014;343:305-309.

15. Fink EC, Krönke J, Hurst SN, et al. Lenalidomide Induces Ubiquitination and Degradation of CSNK1A1 in MDS with Del(5q). ASH Annual Meeting Abstracts. 2014;124.

16. Hsu AK, Quach H, Tai T, et al. The immunostimulatory effect of lenalidomide on NK-cell function is profoundly inhibited by concurrent dexamethasone therapy. Blood. 2011;117:1605-1613.

17. Kumar S, Rajkumar SV. Thalidomide and lenalidomide in the treatment of multiple myeloma. Eur J Cancer. 2006;42:1612-1622.

18. Kastritis E, Dimopoulos MA. The evolving role of lenalidomide in the treatment of hematologic malignancies. Expert Opin Pharmacother. 2007;8:497-509.

19. Richardson PG, Schlossman RL, Weller E, et al. Immunomodulatory drug CC-5013 overcomes drug resistance and is well tolerated in patients with relapsed multiple myeloma. Blood. 2002;100:3063-3067.

20. Weber DM, Chen C, Niesvizky R, et al. Lenalidomide plus dexamethasone for relapsed multiple myeloma in North America. $N$ Engl J Med 2007;357:2133-2142.

21. Dimopoulos M, Spencer A, Attal M, et al. Lenalidomide plus dexamethasone for relapsed or refractory multiple myeloma. $N$ Engl J Med. 2007;357:2123-2132.

22. Dimopoulos MA, Chen C, Spencer A, et al. Long-term follow-up on overall survival from the MM-009 and MM-010 phase III trials of lenalidomide plus dexamethasone in patients with relapsed or refractory multiple myeloma. Leukemia. 2009;23:2147-2152.

23. Stewart AK, Rajkumar SV, Dimopoulos MA, et al. Carfilzomib, lenalidomide, and dexamethasone for relapsed multiple myeloma. $N$ Engl $J$ Med. 2015;372:142-152.

24. Zonder JA, Crowley J, Hussein MA, et al. Lenalidomide and highdose dexamethasone compared with dexamethasone as initial therapy for multiple myeloma: a randomized Southwest Oncology Group trial (S0232). Blood. 2010;116:5838-5841.

25. Rajkumar SV, Jacobus S, Callander NS, et al. Lenalidomide plus highdose dexamethasone versus lenalidomide plus low-dose dexamethasone as initial therapy for newly diagnosed multiple myeloma: an open-label randomised controlled trial. Lancet Oncol. 2010;11:29-37.

26. Benboubker L, Dimopoulos MA, Dispenzieri A, et al. Lenalidomide and dexamethasone in transplant-ineligible patients with myeloma. N Engl J Med. 2014;371:906-917.

27. Attal M, Lauwers-Cances V, Marit G, et al. Lenalidomide maintenance after stem-cell transplantation for multiple myeloma. $N$ Engl J Med. 2012;366:1782-1791.
28. McCarthy PL, Owzar K, Hofmeister CC, et al. Lenalidomide after stemcell transplantation for multiple myeloma. N Engl J Med. 2012;366: $1770-1781$.

29. Palumbo A, Hajek R, Delforge M, et al. Continuous lenalidomide treatment for newly diagnosed multiple myeloma. $N$ Engl J Med. 2012;366: $1759-1769$.

30. Kumar SK, Berdeja JG, Niesvizky R, et al. Safety and tolerability of ixazomib, an oral proteasome inhibitor, in combination with lenalidomide and dexamethasone in patients with previously untreated multiple myeloma: an open-label phase 1/2 study. Lancet Oncol. 2014;15: $1503-1512$.

31. Palumbo A, Anderson K. Multiple myeloma. N Engl J Med. 2011;364: 1046-1060.

32. Corso A, Lorenzi A, Terulla V, et al. Modification of thrombomodulin plasma levels in refractory myeloma patients during treatment with thalidomide and dexamethasone. Ann Hematol. 2004;83:588-591.

33. Zangari M, Saghafifar F, Anaissie E, et al. Activated protein C resistance in the absence of factor $\mathrm{V}$ Leiden mutation is a common finding in multiple myeloma and is associated with an increased risk of thrombotic complications. Blood Coagul Fibrinolysis. 2002;13: 187-192.

34. Palumbo A, Bringhen S, Kumar SK, et al. Second primary malignancies with lenalidomide therapy for newly diagnosed myeloma: a metaanalysis of individual patient data. Lancet Oncol. 2014;15:333-342.

35. Thomas A, Mailankody S, Korde N, Kristinsson SY, Turesson I, Landgren O. Second malignancies after multiple myeloma: from 1960s to 2010s. Blood. 2012;119:2731-2737.

36. Jerez A, Gondek LP, Jankowska AM, et al. Topography, clinical, and genomic correlates of $5 \mathrm{q}$ myeloid malignancies revisited. JClin Oncol. 2012;30:1343-1349.

37. Swerdlow SH, Campo E, Harris NL, et al. WHO Classification of Tumours of Haematopoietic and Lymphoid Tissues. 4th ed. Lyon: IARC; 2008.

38. List A, Dewald G, Bennett J, et al. Lenalidomide in the myelodysplastic syndrome with chromosome 5q deletion. $N$ Engl J Med. 2006;355: $1456-1465$.

39. Fenaux P, Giagounidis A, Selleslag D, et al. A randomized phase 3 study of lenalidomide versus placebo in RBC transfusion-dependent patients with Low-/Intermediate-1-risk myelodysplastic syndromes with del5q. Blood. 2011;118:3765-3776.

40. List A, Kurtin S, Roe DJ, et al. Efficacy of lenalidomide in myelodysplastic syndromes. $N$ Engl J Med. 2005;352:549-557.

41. Raza A, Reeves JA, Feldman EJ, et al. Phase 2 study of lenalidomide in transfusion-dependent, low-risk, and intermediate-1 risk myelodysplastic syndromes with karyotypes other than deletion 5q. Blood. 2008;111:86-93.

42. Sekeres MA, Maciejewski JP, Giagounidis AA, et al. Relationship of treatment-related cytopenias and response to lenalidomide in patients with lower-risk myelodysplastic syndromes. J Clin Oncol. 2008;26: 5943-5949.

43. Fehniger TA, Byrd JC, Marcucci G, et al. Single-agent lenalidomide induces complete remission of acute myeloid leukemia in patients with isolated trisomy 13. Blood. 2009;113:1002-1005.

44. Fehniger TA, Uy GL, Trinkaus K, et al. A phase 2 study of high-dose lenalidomide as initial therapy for older patients with acute myeloid leukemia. Blood. 2011;117:1828-1833.

45. Sekeres MA, Gundacker H, Lancet J, et al. A phase 2 study of lenalidomide monotherapy in patients with deletion $5 \mathrm{q}$ acute myeloid leukemia: Southwest Oncology Group Study S0605. Blood. 2011;118: 523-528.

46. Chen Y, Kantarjian H, Estrov Z, et al. A phase II study of lenalidomide alone in relapsed/refractory acute myeloid leukemia or high-risk myelodysplastic syndromes with chromosome 5 abnormalities. Clin Lymphoma Myeloma Leuk. 2012;12:341-344.

47. Platzbecker U, Germing U. Combination of azacitidine and lenalidomide in myelodysplastic syndromes or acute myeloid leukemia-a wise liaison? Leukemia. 2013;27:1813-1819. 
48. Platzbecker U, Braulke F, Kündgen A, et al. Sequential combination of azacitidine and lenalidomide in $\operatorname{del}(5 \mathrm{q})$ higher-risk myelodysplastic syndromes or acute myeloid leukemia: a phase I study. Leukemia. 2013;27:1403-1407.

49. Garcia-Manero G, Daver NG, Borthakur G, et al. Phase I Study of the Combination of 5-Azacitidine Sequentially with High-Dose Lenalidomide in Higher-Risk Myelodysplastic Syndrome (MDS) and Acute Myelogenous Leukemia (AML). Blood. 2011;118:1122.

50. Pollyea DA, Kohrt HE, Gallegos L, et al. Safety, efficacy and biological predictors of response to sequential azacitidine and lenalidomide for elderly patients with acute myeloid leukemia. Leukemia. 2012;26: 893-901.

51. Pollyea DA, Zehnder J, Coutre S, et al. Sequential azacitidine plus lenalidomide combination for elderly patients with untreated acute myeloid leukemia. Haematologica. 2013;98:591-596.

52. Scherman E, Malak S, Perot C, Gorin NC, Rubio MT, Isnard F. Interest of the association azacitidine-lenalidomide as frontline therapy in highrisk myelodysplasia or acute myeloid leukemia with complex karyotype. Leukemia. 2012;26:822-824.

53. Ramsingh G, Westervelt $P$, Cashen AF, et al. A phase 1 study of concomitant high-dose lenalidomide and 5-azacitidine induction in the treatment of AML. Leukemia. 2013;27:725-728.

54. Sekeres MA, Tiu RV, Komrokji R, et al. Phase 2 study of the lenalidomide and azacitidine combination in patients with higher-risk myelodysplastic syndromes. Blood. 2012;120:4945-4951.

55. Todaro J, Bollmann PW, Rother ET, Giglio AD. Azacitidine and lenalidomide as an alternative treatment for refractory acute myeloid leukemia: a case report. Sao Paulo Med J. In press 2014.

56. Attar EC, Amrein PC, Fraser JW, et al. Phase I dose escalation study of bortezomib in combination with lenalidomide in patients with myelodysplastic syndromes (MDS) and acute myeloid leukemia (AML). Leuk Res. 2013;37:1016-1020.

57. Dennis M, Culligan D, Karamitros D, et al. Lenalidomide monotherapy and in combination with cytarabine, daunorubicin and etoposide for high-risk myelodysplasia and acute myeloid leukaemia with chromosome 5 abnormalities. Leuk Res Rep. 2013;2:70-74.

58. Habermann TM, Lossos IS, Justice G, et al. Lenalidomide oral monotherapy produces a high response rate in patients with relapsed or refractory mantle cell lymphoma. Br J Haematol. 2009;145: 344-349.

59. Zinzani PL, Vose JM, Czuczman MS, et al. Long-term follow-up of lenalidomide in relapsed/refractory mantle cell lymphoma: subset analysis of the NHL-003 study. Ann Oncol. 2013;24:2892-2897.

60. Eve HE, Carey S, Richardson SJ, et al. Single-agent lenalidomide in relapsed/refractory mantle cell lymphoma: results from a UK phase II study suggest activity and possible gender differences. Br J Haematol. 2012;159:154-163.

61. Goy A, Sinha R, Williams ME, et al. Single-agent lenalidomide in patients with mantle-cell lymphoma who relapsed or progressed after or were refractory to bortezomib: phase II MCL-001 (EMERGE) study. J Clin Oncol. 2013;31:3688-3695.

62. Wu L, Adams M, Carter T, et al. lenalidomide enhances natural killer cell and monocyte-mediated antibody-dependent cellular cytotoxicity of rituximab-treated CD20+ tumor cells. Clin Cancer Res. 2008;14: 4650-4657.

63. Wang M, Fayad L, Wagner-Bartak N, et al. Lenalidomide in combination with rituximab for patients with relapsed or refractory mantlecell lymphoma: a phase 1/2 clinical trial. Lancet Oncol. 2012;13: 716-723.

64. Zaja F, De Luca S, Vitolo U, et al. Salvage treatment with lenalidomide and dexamethasone in relapsed/refractory mantle cell lymphoma: clinical results and effects on microenvironment and neo-angiogenic biomarkers. Haematologica. 2012;97:416-422.

65. Flinn IW, Mainwaring M, Peacock N, et al. Rituximab, Lenalidomide, and Bortezomib in the First-Line or Second-Line Treatment of Patients with Mantle Cell Lymphoma a Phase I/II Trial. ASH Annual Meeting Abstracts. 2012;120:2748.
66. Morrison VA, Jung S-H, Johnson J, et al. Salvage Therapy with Bortezomib Plus Lenalidomide for Relapsed/Refractory Mantle Cell Lymphoma: Initial Results of a Phase II Trial (Alliance/CALGB 50501). ASH Annual Meeting Abstracts. 2012;120:3696.

67. Trneny M, Lamy T, Walewski J, et al. Phase II Randomized, Multicenter Study of Lenalidomide Vs Best Investigator's Choice in Relapsed/ Refractory Mantle Cell Lymphoma: Results of the MCL-002 (SPRINT) Study. ASH Annual Meeting Abstracts. 2014;124:626.

68. Ruan J, Martin P, Shah BD, et al. Sustained Remission with the Combination Biologic Doublet of Lenalidomide Plus Rituximab As Initial Treatment for Mantle Cell Lymphoma: A Multi-Center Phase II Study Report. ASH Annual Meeting Abstracts. 2014;124.

69. Wiernik PH, Lossos IS, Tuscano JM, et al. Lenalidomide monotherapy in relapsed or refractory aggressive non-Hodgkin's lymphoma. J Clin Oncol. 2008;26:4952-4957.

70. Witzig TE, Vose JM, Zinzani PL, et al. An international phase II trial of single-agent lenalidomide for relapsed or refractory aggressive B-cell non-Hodgkin's lymphoma. Ann Oncol. 2011;22:1622-1627.

71. Alizadeh AA, Eisen MB, Davis RE, et al. Distinct types of diffuse large B-cell lymphoma identified by gene expression profiling. Nature. 2000;403:503-511.

72. Rosenwald A, Wright G, Chan WC, et al. The use of molecular profiling to predict survival after chemotherapy for diffuse large-B-cell lymphoma. N Engl J Med. 2002;346:1937-1947.

73. Hernandez-Ilizaliturri FJ, Deeb G, Zinzani PL, et al. Higher response to lenalidomide in relapsed/refractory diffuse large B-cell lymphoma in nongerminal center B-cell-like than in germinal center B-cell-like phenotype. Cancer. 2011;117:5058-5066.

74. Zinzani PL, Pellegrini C, Gandolfi L, et al. Combination of lenalidomide and rituximab in elderly patients with relapsed or refractory diffuse large B-cell lymphoma: a phase 2 trial. Clin Lymphoma Myeloma Leuk. 2011;11:462-466.

75. Wang M, Fowler N, Wagner-Bartak N, et al. Oral lenalidomide with rituximab in relapsed or refractory diffuse large cell, follicular and transformed lymphoma: a phase II clinical trial. Leukemia. 2013;27: 1902-1909.

76. Feldman T, Mato AR, Chow KF, et al. Addition of lenalidomide to rituximab, ifosfamide, carboplatin, etoposide (RICER) in first-relapse/ primary refractory diffuse large B-cell lymphoma. Br J Haematol. 2014;166:77-83.

77. Czuczman MS, Davies A, Linton KM, et al. A Phase $2 / 3$ Multicenter, Randomized Study Comparing the Efficacy and Safety of Lenalidomide Versus Investigator's Choice in Relapsed/Refractory DLBCL. ASH Annual Meeting Abstracts. 2014;124.

78. Chiappella A, Tucci A, Castellino A, et al. Lenalidomide plus cyclophosphamide, doxorubicin, vincristine, prednisone and rituximab is safe and effective in untreated, elderly patients with diffuse large B-cell lymphoma: a phase I study by the Fondazione Italiana Linfomi. Haematologica. 2013;98:1732-1738.

79. Vitolo U, Chiappella A, Franceschetti S, et al. Lenalidomide plus R-CHOP21 in elderly patients with untreated diffuse large B-cell lymphoma: results of the REAL07 open-label, multicentre, phase 2 trial. Lancet Oncol. 2014;15:730-737.

80. Nowakowski GS, LaPlant B, Macon WR, et al. Lenalidomide combined with R-CHOP overcomes negative prognostic impact of non-germinal center B-Cell phenotype in newly diagnosed diffuse large B-cell lymphoma: a phase II study. J Clin Oncol. 2015;33(3): 251-257.

81. Reddy NM, Morgan DS, Park SI, Greer JP, Richards KL. Phase II Randomized Study Of Lenalidomide Or Lenalidomide and Rituximab As Maintenance Therapy Following Standard Chemotherapy For Patients With Intermediate-High/High Risk Diffuse Large B-Cell Lymphoma (DLBCL). Blood. 2013;122(21).

82. Tuscano JM, Dutia M, Chee K, et al. Lenalidomide plus rituximab can produce durable clinical responses in patients with relapsed or refractory, indolent non-Hodgkin lymphoma. Br J Haematol. 2014;165: 375-381. 
83. Leonard J, Jung S-H, Johnson JL, et al. CALGB 50401: A randomized trial of lenalidomide alone versus lenalidomide plus rituximab in patients with recurrent follicular lymphoma. ASCO Meeting Abstracts. 2012;30:8000.

84. Fowler NH, Davis RE, Rawal S, et al. Safety and activity of lenalidomide and rituximab in untreated indolent lymphoma: an open-label, phase 2 trial. Lancet Oncol. 2014;15:1311-1318.

85. Kimby E, Martinelli G, Ostenstad B, et al. Rituximab Plus Lenalidomide Improves the Complete Remission Rate in Comparison with Rituximab Monotherapy in Untreated Follicular Lymphoma Patients in Need of Therapy. Primary Endpoint Analysis of the Randomized Phase-2 Trial SAKK 35/10. ASH Annual Meeting Abstracts. 2014;124.

86. Chanan-Khan A, Miller KC, Musial L, et al. Clinical efficacy of lenalidomide in patients with relapsed or refractory chronic lymphocytic leukemia: results of a phase II study. J Clin Oncol. 2006;24: 5343-5349.

87. Ferrajoli A, Lee BN, Schlette EJ, et al. Lenalidomide induces complete and partial remissions in patients with relapsed and refractory chronic lymphocytic leukemia. Blood. 2008;111:5291-5297.

88. Badoux XC, Keating MJ, Wen S, et al. Phase II study of lenalidomide and rituximab as salvage therapy for patients with relapsed or refractory chronic lymphocytic leukemia. J Clin Oncol. 2013;31:584-591.

89. Badoux XC, Keating MJ, Wen S, et al. Lenalidomide as initial therapy of elderly patients with chronic lymphocytic leukemia. Blood. 2011;118: 3489-3498.

90. Chen CI, Bergsagel PL, Paul H, et al. Single-agent lenalidomide in the treatment of previously untreated chronic lymphocytic leukemia. J Clin Oncol. 2011;29:1175-1181.

91. James DF, Werner L, Brown JR, et al. Lenalidomide and rituximab for the initial treatment of patients with chronic lymphocytic leukemia: a multicenter clinical-translational study from the chronic lymphocytic leukemia research consortium. J Clin Oncol. 2014;32:2067-2073.
92. Zinzani PL, Pellegrini C, Broccoli A, et al. Lenalidomide monotherapy for relapsed/refractory peripheral T-cell lymphoma not otherwise specified. Leuk Lymphoma. 2011;52:1585-1588.

93. Morschhauser F, Fitoussi O, Haioun C, et al. A phase 2, multicentre, single-arm, open-label study to evaluate the safety and efficacy of single-agent lenalidomide (Revlimid) in subjects with relapsed or refractory peripheral T-cell non-Hodgkin lymphoma: the EXPECT trial. Eur J Cancer. 2013;49:2869-2876.

94. Hopfinger G, Nösslinger T, Lang A, et al. Lenalidomide in combination with vorinostat and dexamethasone for the treatment of relapsed refractory peripheral T cell lymphoma (PTCL): report of a phase I/II trial. Ann Hematol. 2014;93:459-462.

95. Fehniger TA, Larson S, Trinkaus K, et al. A phase 2 multicenter study of lenalidomide in relapsed or refractory classical Hodgkin lymphoma. Blood. 2011;118:5119-5125.

96. Revicki DA, Brandenburg NA, Muus P, Yu R, Knight R, Fenaux P. Health-related quality of life outcomes of lenalidomide in transfusiondependent patients with Low- or Intermediate-1-risk myelodysplastic syndromes with a chromosome $5 \mathrm{q}$ deletion: results from a randomized clinical trial. Leuk Res. 2013;37:259-265.

97. Dimopoulos MA, Delforge M, Hájek R, et al. Lenalidomide, melphalan, and prednisone, followed by lenalidomide maintenance, improves health-related quality of life in newly diagnosed multiple myeloma patients aged 65 years or older: results of a randomized phase III trial. Haematologica. 2013;98:784-788.

98. Delforge M, Minuk L, Eisenmann J-C, et al. Health-related quality of life (HRQOL) in transplant-ineligible patients (pts) with newly diagnosed multiple myeloma (NDMM): Results from the first trial. $A S C O$ Meeting Abstracts. 2014;32:8516.
Cancer Management and Research

\section{Publish your work in this journal}

Cancer Management and Research is an international, peer-reviewed open access journal focusing on cancer research and the optimal use of preventative and integrated treatment interventions to achieve improved outcomes, enhanced survival and quality of life for the cancer patient The journal welcomes original research, clinical \& epidemiological

\section{Dovepress}

studies, reviews \& evaluations, guidelines, expert opinion \& commentary, case reports \& extended reports. The manuscript management system is completely online and includes a very quick and fair peerreview system, which is all easy to use. Visit http://www.dovepress.com/ testimonials.php to read real quotes from published authors. 